\title{
Contrast-increment thresholds are related to variability in the apparent contrast function
}

\author{
JOHN A. BARO \\ Washington University, St. Louis, Missouri \\ and \\ STEPHEN LEHMKUHLE and RAYMOND A. APPLEGATE \\ University of Missouri-St. Louis, St. Louis, Missouri
}

\begin{abstract}
Although Fechner (1860/1912) asserted that the gain of a sensory system should determine its ability to resolve different stimulus magnitudes, most researchers have found that gain and resolving power are not consistently related. The present study assessed gain (measured by magnitude estimation) and increment threshold (measured by a forced-choice procedure) in the same observers under the same viewing conditions. It was found that gain and increment threshold were not inversely related, as proposed by Fechner. However, relative variability in apparent contrast (standard error/magnitude estimate) was correlated with relative increment threshold (increment threshold/background contrast). This correlation was even higher following pattern adaptation. These findings imply that resolving power for contrast is determined by variability in the system's response to contrast rather than by its gain. Because this relationship is strengthened by adaptation, adaptation may reduce the contribution of other factors that add noise to the unadapted visual system.
\end{abstract}

Fechner (1860/1912) asserted that the rate at which apparent stimulus magnitude changes with the physical magnitude of a stimulus determines a sensory system's ability to resolve different stimulus magnitudes. Since that time, there has been considerable controversy regarding the general validity of Fechner's assertion. For some prothetic continua Fechner's law has been confirmed (e.g., Mansfield, 1976, for brightness). ${ }^{1}$ However, in most cases, apparent magnitude and resolving power (i.e., difference threshold) are not consistently related (e.g., see Stevens, 1961b). In contrast perception, a systematic relation between measures of gain (i.e., in this context, the slope of the apparent vs. physical contrast function) and resolving power (i.e., contrast difference threshold as a function of contrast) has been difficult to establish. Differences in experimental procedures and dependent measures across studies have resulted in a number of conflicting results.

In the assessment of contrast gain, some investigators have reported that the relation between physical and perceived contrast is best described by a power function (e.g., Franzen \& Berkley, 1975; Gottesman, Rubin, \& Legge, 1981), whereas others have found this function to be linear under some circumstances (Biondini \& deMattiello, 1985; Cannon, 1979). High-contrast pattern adaptation can affect the gain function by reducing the apparent contrast of subsequently viewed suprathreshold patterns. Kulikowski (1976) reported an overall lowering of the ap-

Correspondence should be addressed to John A. Baro, School of Optometry, University of Missouri-St. Louis, St. Louis, MO 63121. parent contrast function; however, the adapted functions were still exponential, with slopes similar to those of unadapted functions (i.e., gain was unchanged). Blakemore, Muncey, and Ridley (1971) found that while the apparent contrast of all test patterns decreased following adaptation, the effect varied with contrast of the test pattern. The result was a power function with an increased slope (i.e., higher gain). Because of the increase in gain, Blakemore et al. predicted an improvement in resolving power following adaptation, which is consistent with Fechner's assertion (i.e., gain determines resolving power).

In the assessment of contrast difference thresholds, Kohayakawa (1972) found that the increment threshold for sine-wave gratings was highest at an intermediate base contrast, and decreased for higher and lower base contrasts. To the contrary, Bodis-Wollner, Hendley, and Kulikowski (1972) reported that increment threshold monotonically decreased over the same range of base contrasts studied by Kohayakawa. Still others (Burton, 1981; Carlson \& Pica, 1979; Foley \& Legge, 1981; Nachmias \& Sansbury, 1974; Swift \& Smith, 1984) have reported that increment threshold is lowest for base contrasts near the detection threshold and increases at higher and lower base contrasts. Despite these inconsistencies, it is generally agreed that contrast-increment threshold increases monotonically with suprathreshold base contrast.

The prediction of a decreased increment threshold resulting from increased gain following adaptation has not been confirmed. Kulikowski (1976) found that highcontrast adaptation slightly increased increment thresholds. Legge (1981) reported that increment thresholds for 8-cycle per degree (cpd) patterns increased 
only when the contrast of the adapting pattern was high and the base contrast of the test pattern was low. The increment threshold of 2-cpd patterns was unaffected by adaptation. Barlow, Macleod, and van Meeteren (1976) found either no change in increment thresholds or a slight increase only when the contrast of the adapting pattern was high. Both Legge and Barlow et al. reported that effects of adaptation were greatest at low base contrasts and effects were negligible for higher base contrasts. These findings are contrary to what would be predicted on the basis of Fechner's assertion. ${ }^{2}$

Procedural differences among the above studies precludes reconciliation of the conflicting observations. Hence, it is impossible to confirm or deny the relation between contrast gain and resolving power proposed by Fechner. The purpose of the present study was to determine whether or not a relation between gain and increment threshold exists by testing the same observers in two experimental conditions. In Experiment 1 both adapted and unadapted increment thresholds were measured as a function of base (background) contrast. In Experiment 2 apparent contrast was measured as a function of physical contrast in the same observers under the same viewing conditions. If increment threshold and gain were mediated by the underlying process proposed by Fechner, then an inverse relation between the two measures would be expected and both should be similarly affected by adaptation.

\section{GENERAL METHOD}

\section{Stimulus Generation}

Stimuli were generated on a Tektronix 608 monitor (P31 phosphor). An Apple II computer was used to control the display. The monitor screen $(10 \times 12 \mathrm{~cm})$ was masked down to a circular area with a diameter of $9 \mathrm{~cm}$. When viewed from a distance of $100 \mathrm{~cm}$, the mask subtended $5.14^{\circ}$ of visual angle. Mean luminance of the display was held constant at $44.7 \mathrm{~cd} / \mathrm{m}^{2}$.

Vertical grating patterns, sinusoidally modulated in luminance, were updated at $200 \mathrm{~Hz}$ from digital waveform tables stored in the computer's memory. The waveforms had a mean of zero so that the average luminance of the display was identical to that of the unmodulated raster. Spatial frequency of the patterns could be varied from 0.6 to 10 cycles per degree (cpd) at the viewing distance of $1 \mathrm{~m}$. Contrast was varied only over the range in which the relation of the $z$-axis voltage to luminance was linear, from 0.0 to 0.53 , where contrast is defined as the difference between the maximum and minimum luminance divided by their sum. All patterns used for adaptation were counterphased (square-wave) at either 1 or $8 \mathrm{~Hz}$.

\section{Observers}

One of the observers, author J.B., was experienced with grating patterns and psychophysical procedures. Measurements were also obtained from 3 naive, inexperienced observers. All observers had corrected acuities of 20/20 or better and were free from ocular or systemic pathology. Natural pupils and spectacle corrections were used throughout. All stimuli were viewed monocularly with the dominant eye (sighting dominance); the contralateral eye was covered with an opaque occluder.

Each observer was seated with his/her head held steady by a chin and forehead rest. The only source of illumination during data collection was the CRT display. Threshold responses were made on a small keypad placed within easy reach of the observer. Responses for the magnitude estimation procedure were verbal and entered by the experimenter at the computer's keyboard. All responses were recorded by the computer and stored on floppy diskettes.

\section{EXPERIMENT 1}

\section{Procedure}

Two-alternative, temporal forced-choice trials in conjunction with a staircase procedure were used to measure contrast-increment thresholds as a function of base contrast. Four minutes of preadaptation preceded each trial block. Each trial consisted of $5 \mathrm{sec}$ of readaptation, followed by two 250 -msec observation intervals, indicated by tones. Each observation interval was preceded by $250 \mathrm{msec}$ of a homogeneous screen. One of the observation intervals contained a signal grating (base contrast plus an increment), and the other contained a grating of lower contrast (the base contrast). The signal was presented randomly in the first or second interval with equal probability. The observer's task was to indicate the interval containing the signal. The observer received feedback in the form of coded tones immediately following every response. Each response initiated the next trial.

The staircase procedure was used to determine the minimum contrast increment that could be discriminated at a $70 \%$ correct level (see Wetherhill \& Levitt, 1965). Initially, the staircase moved in relatively large steps (the size of the first step varied from 0.017 for low base contrasts up to 0.050 for the highest base contrast). Following the 2nd, 4th, 6th, and 8 th reversals, the step size was reduced by one-half. The staircase terminated after 15 reversals had occurred. Contrast-increment threshold was defined as the (arithmetic) mean contrast increment associated with the last 7 reversals.

Each adapting interval consisted of either a homogeneous screen or a sine-wave grating pattern. Adapting patterns were alternated in counterphase at either 1 or $8 \mathrm{~Hz}$, and their contrast was one of the following: 0.0 (unadapted condition), $0.133,0.265$, or 0.530 . A fixation mark centered on the screen was used to assist the observer in maintaining steady fixation. Test patterns were always stationary, and their base contrast was one of the following: 0.0 (detection threshold), $0.03,0.06,0.12,0.24$, or 0.48 . Spatial frequency of the test and adapting patterns was $1,2,5$, or $10 \mathrm{cpd}$. Test and adapting patterns always had the same spatial frequency. Increment thresholds for each of the test conditions were obtained from Observers J.B., L.P.A., and B.K.T.; detection thresholds only (base contrast of zero) were obtained from Observer L.E.

\section{Results}

Figure 1 shows the mean unadapted increment threshold as a function of base contrast at $1,2,5$, and $10 \mathrm{cpd}$. Detection thresholds (not shown) were consistently higher than increment thresholds at the lowest base contrast. This result is in agreement with reports of a dipper-shaped function for contrast-increment threshold (e.g., Bradley \& Ohzawa, 1986; Carlson \& Pica, 1979). Regression analyses suggest that the relation between base contrast and increment threshold is not consistently fit by either a linear function or a power function across subjects or stimulus conditions.

Although detection thresholds consistently increased following adaptation, mean increment threshold across observers remained largely unaffected by adaptation. The results are different, however, when the data are examined for each observer individually. Figure 2 illustrates the extent of individual differences in the adapted increment 


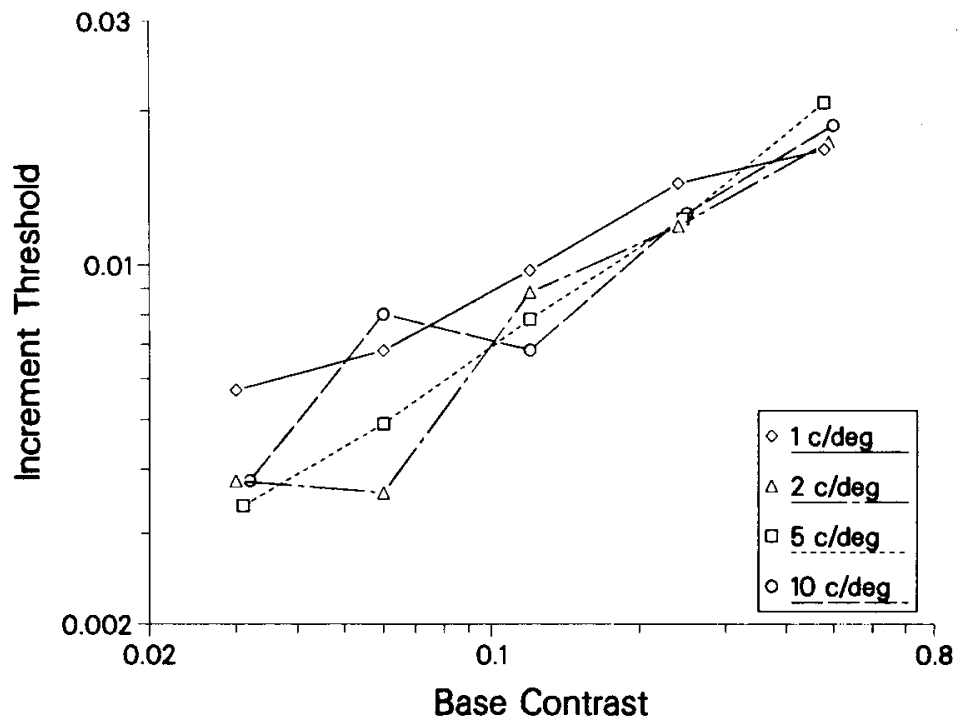

Figure 1. Mean unadapted increment thresholds as a function of base contrast for Observers B.K.T., J.B., and L.P.A. Each data point is based on three staircases per observer. Spatial frequencies are indicated in the legend. Detection thresholds (i.e., a base contrast of 0 ; not shown) were consistently higher than increment thresholds at the lowest base contrast.

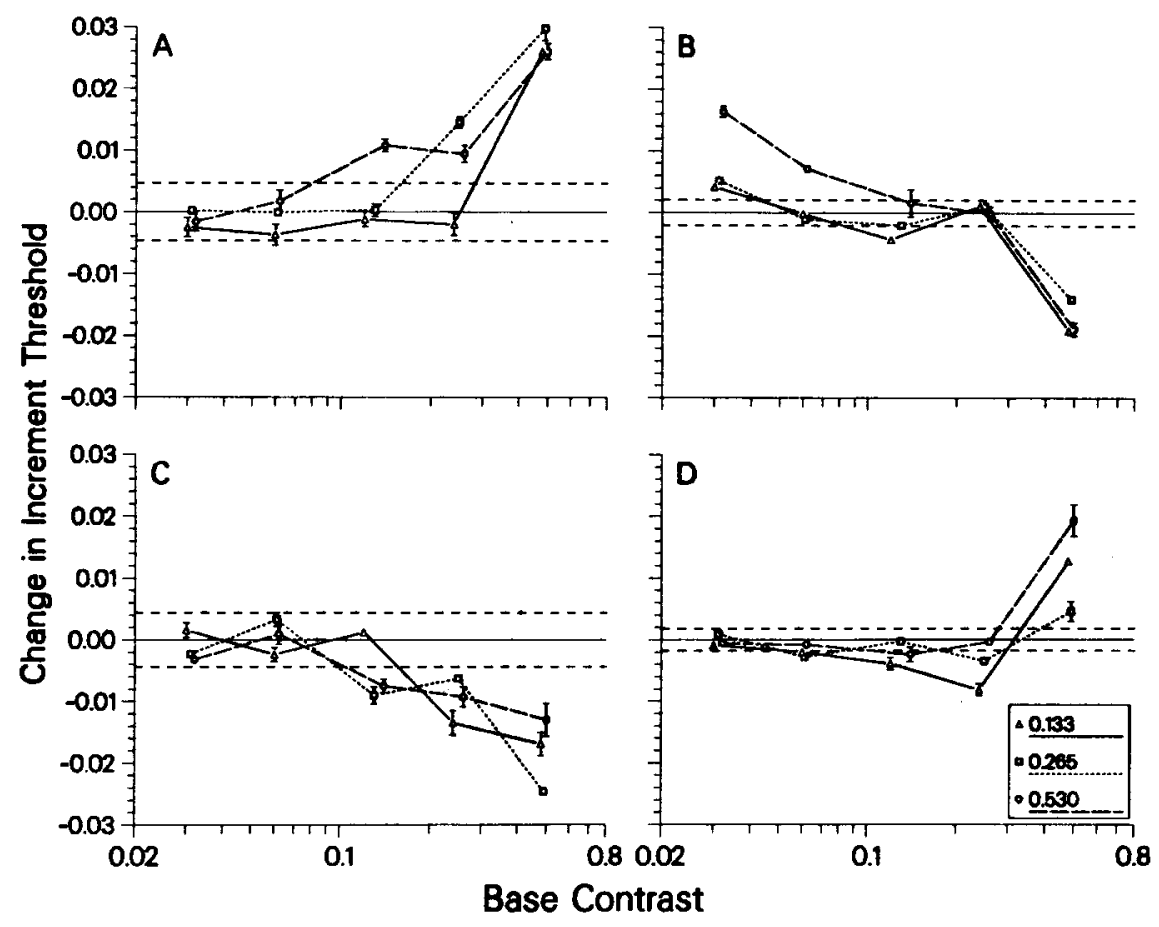

Figure 2. Change in increment threshold following adaptation for individual observers. Adapting contrasts are indicated in the legend. A positive value indicates an increase in increment threshold following adaptation, and a negative value indicates a decrease. Error bars indicate \pm 1 standard error of the mean. Horizontal dashed lines represent confidence intervals for redetermining unadapted increment threshold (after DeValois, 1977). The confidence intervals were calculated by taking the mean standard error for each curve (i.e., the sum of 5 standard error values divided by 5 ) and multiplying this value by 3 . This range corresponds to a confidence interval of approximately 99.5\%. (A) Observer L.P.A., 1-cpd, 1-Hz adapting pattern; (B) Observer J.B., 1-cpd, 8Hz adapting pattern; (C) Observer L.P.A., 2-cpd, 8-Hz adapting pattern; (D) Observer B.K.T., 2-cpd, 8-Hz adapting pattern. 
threshold function. Significant effects of adaptation were obtained in some cases, but these changes were not consistent across conditions or observers and therefore tended to cancel out when the data were averaged. Overall, changes in increment threshold following adaptation were greatest for the lowest and highest base contrasts, and the most common effect was an increase in increment threshold. It should also be pointed out that in some cases increment threshold decreased following adaptation (see Figure 2, B and C). However, neither magnitude nor direction of the effect appears to be influenced by spatial frequency, temporal frequency, or contrast of the adapting pattern.

\section{Discussion}

Changes in increment threshold following adaptation were generally small and inconsistent. In many ways, the results reported here are similar to those obtained elsewhere with comparable procedures. Both Barlow et al. (1976) and Legge (1981) reported either no change or slight increases (less than $20 \%$ ) in increment thresholds following adaptation. Legge also noted that the effects of adaptation appear smaller when measured with a forcedchoice procedure than when the method of adjustment is used. This observation suggests that the primary effect of adaptation may be on the observer's response criterion rather than on his/her ability to discriminate contrast.

Some differences between the present findings and others are also apparent. First, although both Legge (1981) and Barlow et al. (1976) found that the effects of adaptation were greatest at low base contrasts, increment thresholds reported here changed at both low and high base contrasts. Second, Barlow et al. reported no effect when the adapting contrast was less than or equal to the base contrast of the test pattern. For the data reported here, changes in increment threshold for both high and low base contrasts were independent of adapting contrast. Third, Legge found that the effects of adaptation varied with spatial frequency; no comparable spatial frequency effect was found here.

A number of procedural differences may account for some of these conflicting results, and the most relevant appears to be differences in adapting procedure. The use of stationary adapting patterns generally results in larger aftereffects (see, e.g., DeValois, 1977; Legge, 1981) than those measured following adaptation to counterphased patterns (Barlow et al., 1976; this trend was also evident in pilot data not reported here). Individual differences also appear to play a prominent role in the way data are interpreted. Although data plotted for individual observers are similar in many respects to previously reported results, large effects tended to cancel out when the data were averaged across observers. Mean changes in increment threshold rarely exceeded 15\%; however, individual thresholds at the highest and lowest base contrasts changed by as much as $50 \%$.

\section{EXPERIMENT 2}

\section{Procedure}

A magnitude estimation task was used to assess apparent contrast as a function of physical contrast. Before each trial block began, a grating pattern having a spatial frequency of $1 \mathrm{cpd}$ and a contrast of 0.157 was displayed for $5 \mathrm{sec}$. Observers were informed that this pattern was the standard and that it should be assigned the value 100 (the modulus). The same standard was used for all test conditions. Four minutes of preadaptation followed presentation of the standard. Each trial consisted of $5 \mathrm{sec}$ of readaptation, followed by a 1-sec observation interval containing the test pattern and indicated by a tone. Any time that elapsed between the end of the observation interval and the beginning of the next trial (i.e., the time necessary to record the observer's verbal response) was filled with the adapting pattern.

Each trial block consisted of $\mathbf{4 0}$ trials, all devoted to test patterns of the same spatial frequency. Spatial frequency of both the test and adapting patterns was $1,2,5$, or $10 \mathrm{cpd}$. Five estimates were made for each of eight contrasts $(0.030,0.047,0.070,0.105,0.157$, $0.235,0.353$, and 0.530 ) during each trial block. The arithmetic mean of these five estimates was defined as the observer's estimate of the contrast of that pattern. The order of presentation of the test patterns was randomized within each trial block. Between trial blocks, the temporal frequency and contrast of the adapting pattern were varied among the same values used in Experiment 1. Magnitude estimates were obtained from Observers L.P.A., B.K.T., and L.E. for all conditions. All other testing conditions were identical to those specified for Experiment 1.

In addition, the observers were tested under two other conditions. The first of these was used to assess the validity of the adaptation effects. Conditions for these trial blocks were identical to those mentioned above, with one exception: the spatial frequency of adapting and test patterns was not the same. The second condition was used to assess range effects and the utility of a threshold correction. The same number of test contrasts were used; however, their spacing was increased, resulting in an increase in the overall range of contrast within a trial block. Contrast ranges of 0.03-0.53 (1.26 $\log$ units) and 0.004-0.52 (2.11 log units) were compared under otherwise similar conditions.

\section{Results}

Mean unadapted magnitude estimates of 1-, 2-, 5-, and 10-cpd patterns are shown in Figure 3. The slopes (i.e., the exponents of the power functions) for the curves are $0.354,0.246,0.210$, and 0.246 , respectively. Since observers were provided with a modulus, the data have not been scaled. Both mean slope and slopes for individual observers varied as a function of spatial frequency, being lowest at $5 \mathrm{cpd}$ and increasing at higher and lower spatial frequencies. It should also be pointed out that slope varied considerably between observers. Observer B.K.T. consistently had the highest slope (0.37-0.51), Observer L.P.A. consistently had the lowest slope (0.09-0.23), and Observer L.E. was in between (0.11-0.40).

The magnitude of the slopes deserves additional attention. For similar procedures, reported slopes typically range from 0.5 to well over 1.0 (Cannon, 1984; Franzen \& Berkley, 1975; Gottesman, Rubin, \& Legge, 1981). One possible cause of this difference in slope is the range of contrasts used in the present study. To assess the ef- 


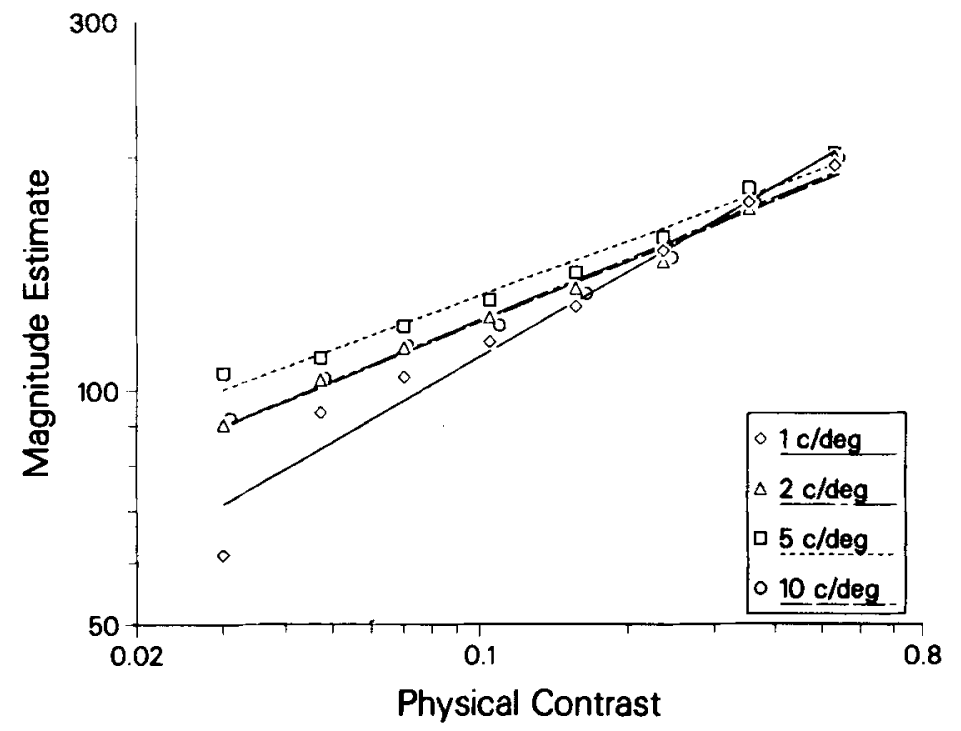

Figure 3. Mean unadapted magnitude estimates as a function of physical contrast for Observers B.K.T., L.E., and L.P.A. across all spatial and temporal frequencies. Spatial frequencies are indicated in the legend. The lines drawn through the points have been fitted by the least squares criterion.

fect of range on slope, we obtained magnitude estimates for a larger range of contrasts. The results of this manipulation are given in Figure 4, which shows that over the portion of the contrast range at which the two curves overlap, estimates are virtually identical. However, below a contrast of 0.03 , estimates of magnitude drop off sharply, as would be expected when measurements of any prothetic continuum are made near threshold (Stevens, 1961a). This is in contrast to the typically reported range effects that indicate that slope generally decreases with increases in stimulus range (e.g., see Cannon, 1984). We have no explanation for the consistently low slopes obtained from these observers or for the lack of a consistent range effect.

The effects of adaptation on mean magnitude estimate are illustrated in Figure 5. Effects were qualitatively similar under all conditions for all observers. Several general trends in these data are apparent: The change in magnitude estimate increased with the contrast of the adapting pattern. Change in magnitude estimate was greater at lower physical contrasts than at higher contrasts. For physical contrasts equal to or higher than the adapting contrast, there was virtually no effect. As a result of this differential effect of adaptation (as a function of physical contrast), there tended to be a sharp drop in apparent contrast near the low end of the contrast range so that adapted functions were concave downward. In many cases, apparent contrast decreased sufficiently that low-contrast patterns were no longer visible (i.e., they received estimates of zero). Analyses of variance and regression analyses indicated significant changes in the slope and the

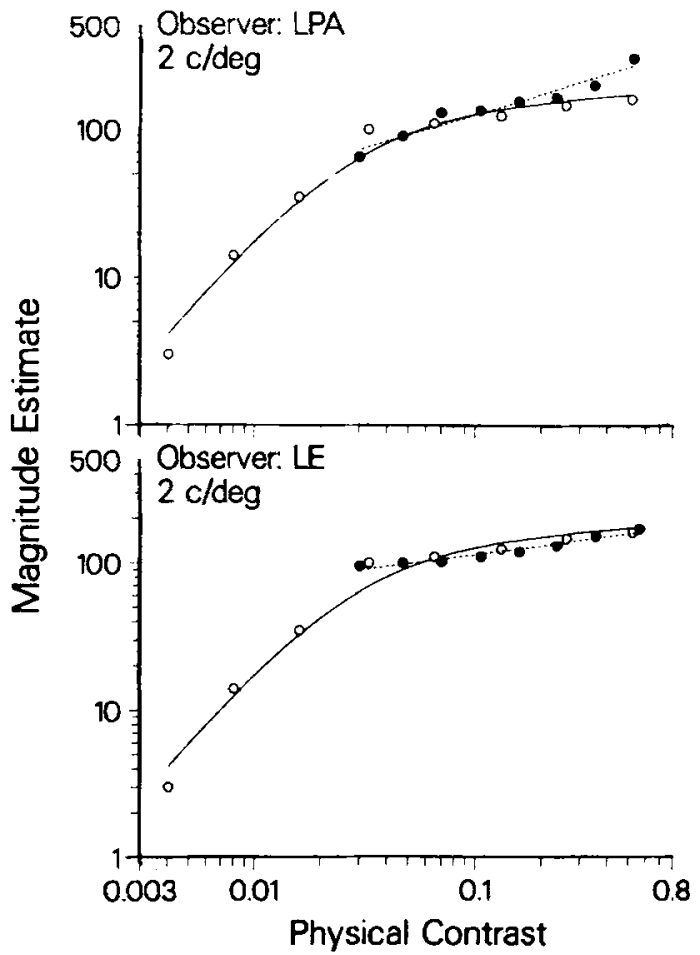

Figure 4. Magnitude estimation functions typical of those obtained with the longer contrast range. Each point represents the mean of a single magnitude estimation procedure. Filled symbols represent the normal range (1.26 log units), and open symbols represent the extended range (2.11 $\mathrm{kg}$ units). Curves have been fitted using a parametric spline smonthing function. Observers and spatial frequencies are indicated in the figure. 


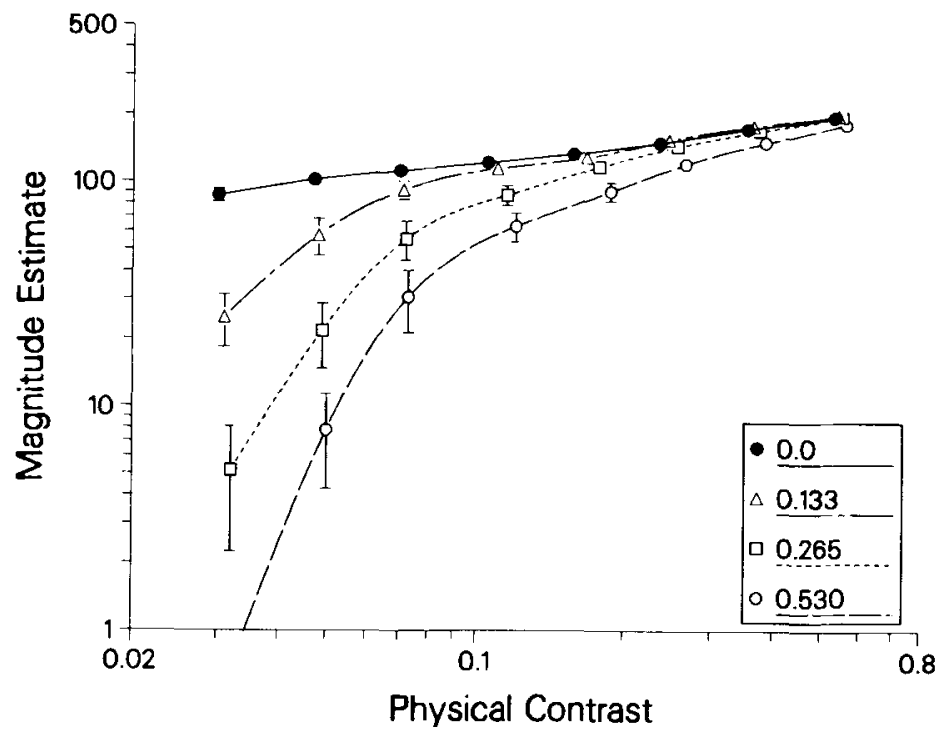

Figure 5. Mean magnitude estimates with and without adaptation for Observers B.K.T., L.E., and L.P.A. across all spatial and temporal frequencies. Adapting contrasts are indicated in the legend; filled symbols indicate the unadapted condition. Curves have been fitted using a parametric spline smoothing function. Curves that extend past the bottom of the figure indicate magnitude estimates of zero below that point. Error bars indicate \pm 1 standard error of the mean.

overall shape of virtually all individual functions obtained following high-contrast adaptation.

To confirm the validity of the adaptation effects and to demonstrate their spatial frequency specificity, we obtained magnitude estimates when the spatial frequency of the test and adapting patterns differed. The data obtained from these conditions are presented in Figure 6. Analyses of variance indicated no significant differences between unadapted magnitude estimates and those made following adaptation to a pattern that differed from the test pattern by two or more octaves.

It has been noted that magnitude estimates of stimuli near detection threshold tend to decrease suddenly. Since detection thresholds were consistently raised following adaptation, it might be possible to account for the steep portion of the adapted functions by an increased detection threshold. To determine whether change in detection threshold could account for the shape of the adapted magnitude estimation functions, we applied a threshold correction to the mean function obtained following adaptation to a contrast of 0.53 (i.e., mean adapted detection threshold was subtracted from each physical contrast value). This threshold correction is believed to be useful in bringing the zero of the physical scale into agreement with the zero of the psychological scale (Stevens, 1961a). The result of this manipulation is presented in Figure 7. For the mean and virtually all individual conditions, $r^{2}$ increased and slope decreased when the functions were corrected. Although the corrected curves more closely approximate power functions, they are clearly not straight lines on $\log$-log coordinates. This suggests that the shape

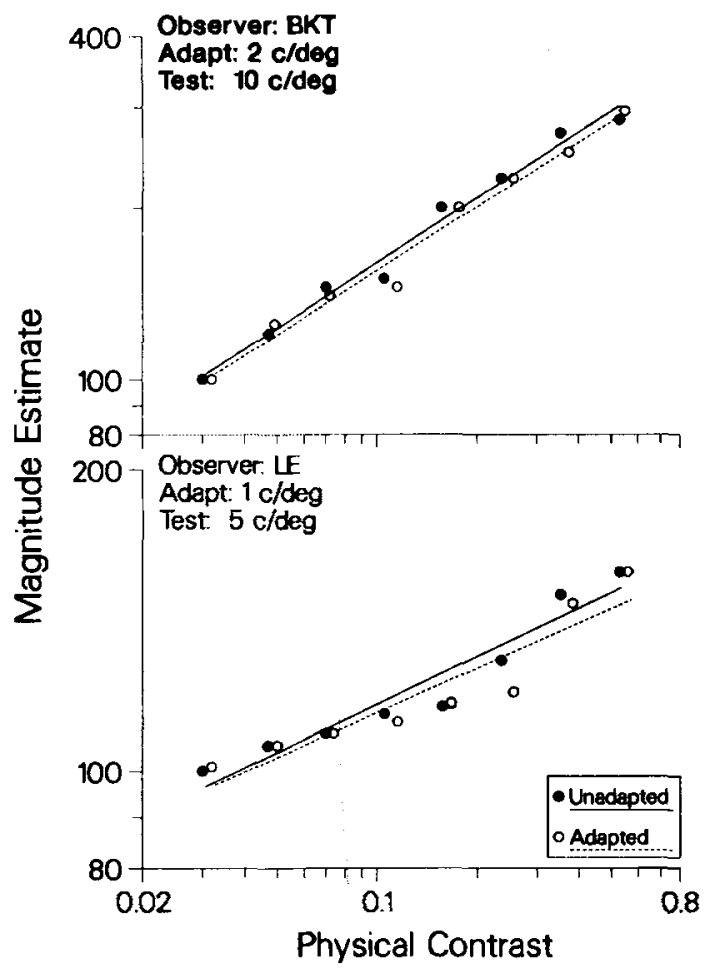

Figure 6. Representative magnitude estimation functions typical of those obtained when the spatial frequency of the adapting and test patterns differed. Lines drawn through the points have been fitted by the least squares criterion. The temporal frequency of adapting patterns was $1 \mathrm{~Hz}$. Observers and spatial frequencies are indicated in the figure. 


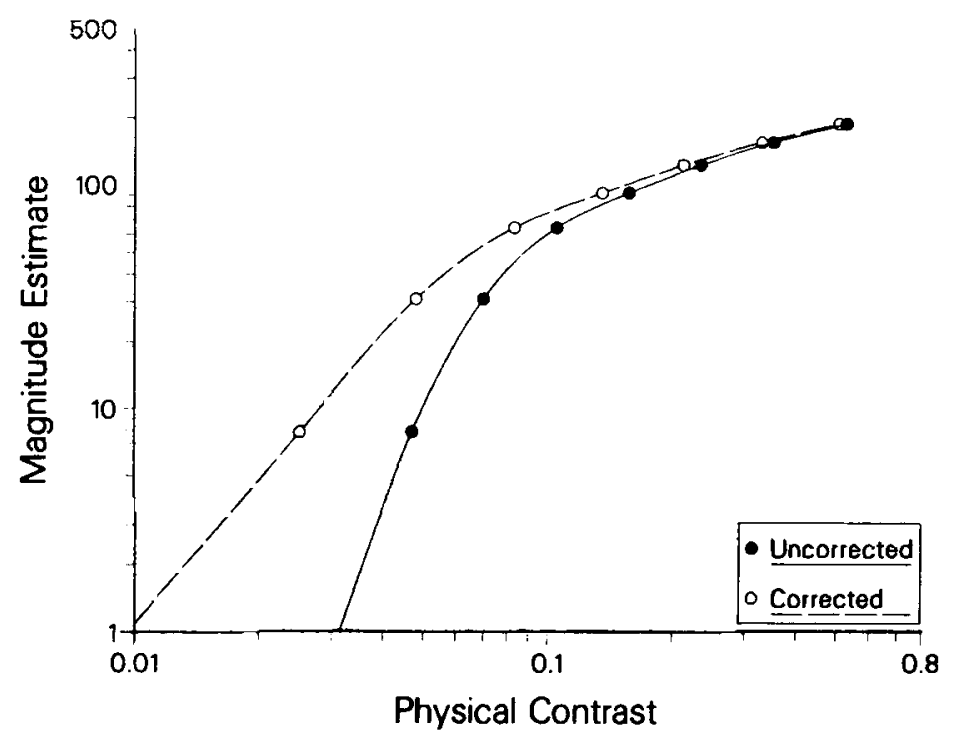

Figure 7. Mean magnitude estimation functions for Observers B.K.T., L.E., and L.P.A. following high-contrast (0.53) adaptation at all spatial and temporal frequencies. Open symbols represent the result of a threshold correction (i.e., the mean detection threshold has been subtracted from the physical contrast value for each point). Curves have been fitted using a parametric spline smoothing function. Curves that extend past the bottom of the figure indicate magnitude estimates of zero below that point.

of the adapted apparent contrast functions cannot be accounted for entirely by an increase in detection threshold. Factors other than change in detection threshold must therefore contribute to the change in apparent contrast following adaptation.

\section{Discussion}

Contrast gain is defined here as the slope of the function derived from magnitude estimation. Although some investigators have reported that perceived contrast increases linearly with physical contrast (e.g., Cannon, 1979; Fiorentini \& Maffei, 1973), the present data and other investigators (e.g., Franzen \& Berkley, 1975; Gottesman et al., 1981) suggest a power function relationship. Since most deviations from the power function occur when magnitude estimates are obtained near threshold, this inconsistency appears to be a range effect.

Although adaptation typically results in an overall decrease in apparent contrast, the effects on contrast matches are qualitatively different from the effects on magnitude estimates. Blakemore et al. (1971) found that the slope of adapted matching functions consistently increased, but the functions were still straight lines on log-log coordinates. Likewise, Kulikowski (1976) fitted his adapted functions, obtained using both contrast matching and magnitude production, with straight lines. Concave adapted functions similar to those reported here have been reported elsewhere only for other sensory continua (e.g., Mansfield, 1976, for adapted brightness functions; Stevens, 1958, for adapted loudness matches).
In other sensory continua, concave adapted functions are normally associated with an increase in detection threshold, and the threshold correction is often used to remove the concavity. In the case of perceived contrast, however, changes observed following adaptation cannot be accounted for solely in terms of an elevated detection threshold. These discrepancies can best be explained by differences in adapting procedures. Data were also collected using stationary adapting patterns (not reported here), and the results were comparable to those reported by Blakemore et al. (1971); that is, slope increased following adaptation, but the adapted functions were still exponential. It seems likely that the interpretation of results may be complicated by factors such as afterimages, eye movements, or phase differences when stationary adapting patterns are used. A systematic investigation of these differential effects is needed to clarify this issue.

\section{GENERAL DISCUSSION}

Fechner proposed that gain (the slope of the apparent vs. physical contrast function) and resolving power were inversely related (i.e., a steeper slope would result in a lower increment threshold). Our results indicate that the apparent contrast function cannot be predicted from increment threshold data in the way Fechner suggested. Figure 8A shows mean increment threshold as a function of gain (i.e., slope of the magnitude estimation function) for all observers at each spatial frequency. Fechner's model predicts an inverse relation between the two mea- 

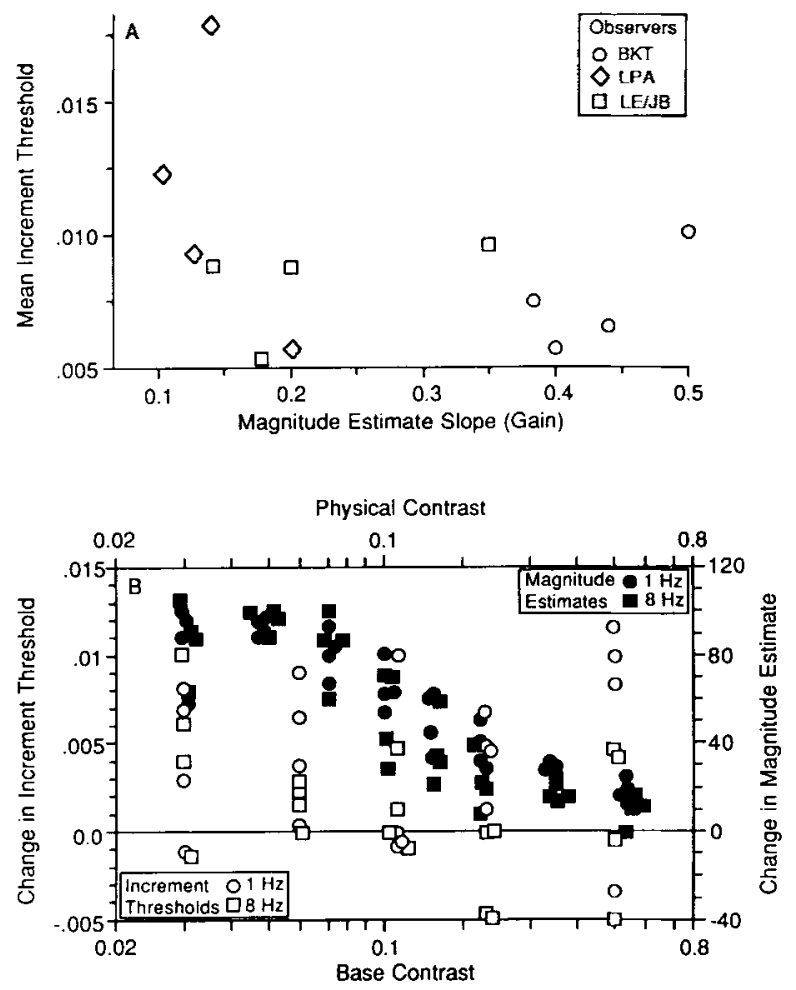

Figure 8. (A) Mean increment threshold versus slope of the corresponding magnitude estimation function (unadapted). Each data point is the mean across spatial frequencies for an individual observer. Because Observers L.E. and J.B. did not participate in both experiments, their data have been combined. Fechner's (1860/1912) law would predict an inverse relation between the two measures (i.e., a negative slope). (B) Change in magnitude estimate and increment threshold following high-contrast adaptation as a function of contrast. Each data point is the mean of all observers at an individual spatial and temporal frequency. Increment threshold data points (open symbols) correspond to the bottom and left axes. Magnitude estimation data points (filled symbots), which correspond to the top and right axes, have been multiplied by -1 . As a result of this transformation, the vertical axes for both sets of data indicate the amount by which sensitivity decreased following adaptation. (An increase in increment thresbold indicates a decrease in sensitivity; a decrease in magnitude estimate indicates a decrease in sensitivity.)

sures, and this is clearly not the case $(r=-.330$, $p>$.29). The lack of a simple relation between these two measures becomes more apparent when the effects of adaptation are considered. Figure $8 \mathrm{~B}$ illustrates the effect of high-contrast adaptation on increment thresholds and magnitude estimates. Although both measures indicate changes in sensitivity following adaptation, the effect of adaptation on each of these measures is different. Change in magnitude estimate consistently decreases with increasing contrast $(r=-.832, p<.001)$, but changes in increment threshold are inconsistent with respect to contrast, spatial frequency, and temporal frequency of the adapting pattern $(r=.075, p>.63)$. The implication is that these two psychophysical measures do not reveal the underlying mechanism implied by Fechner's proposal.

The finding that increment thresholds and magnitude estimates (on a prothetic continuum) are not consistently related is by no means a new one. Nevertheless, the intuitive appeal of the simple model proposed by Fechner still pervades modern psychophysics, even though this principle has been shown to be inappropriate. According to Stevens (1957), the fundamental problem with Fechner's assertion lies in the assumption that the perceived just noticeable difference (JND) remains constant across changes in stimulus magnitude. The JND can be thought of as a measurement of dispersion or error, and, rather than being constant, the JND tends to grow with subjective magnitude. This function has been quantified by Stevens (1957), who found that the subjective size of the JND grows as an exponential function of the number of JNDs above threshold. Although this finding provides a compelling reason to doubt the possibility of ever finding the relationship suggested by Fechner, it does, however, suggest another possibility.

If the increment threshold is a measure of error, then it seems reasonable to expect that increment threshold may be related to variability in the apparent contrast function. To test this prediction, variability (indicated by the standard error of the mean, SEM) was assessed as a function of physical contrast. Although increment threshold was found to increase monotonically with base contrast, mean SEM tended to remain fairly constant over the range of contrasts studied here (for 2 observers there was a slight decrease in SEM as physical contrast increased). Therefore, it is apparent that increment threshold and variability are not related in such a straightforward manner.

Another means of assessing variability is as a relative measure; that is, it is sometimes more meaningful to examine variability relative to its mean. On a number of sensory continua, it has been shown that while absolute variability increases with the mean, relative variability tends to remain constant or decrease (Stevens, 1957). The most commonly used measure of relative variability is the coefficient of variation, defined as the standard deviation divided by the mean. This measure of variability is not new to psychophysics; a comparable measure of increment threshold, the Weber fraction, has been in use for many years. To determine whether an assessment of relative variability would prove more useful than the absolute measure, we analyzed variability relative to the appropriate mean. As a measure of relative increment threshold, the Weber fraction (increment threshold/base contrast) was used. Relative variability of the apparent contrast function was derived by dividing the SEM by the mean magnitude estimate. The results of this analysis, across subjects and conditions, are presented in Figure 9 and Table 1. Similar results were obtained for individual observers at all spatial and temporal frequencies tested. It is obvious from both the figure and the table that there is a correlation between the relative measures of increment threshold and SEM, with and without adaptation.

Several features of Figure 9 merit attention. First, all curves have a negative slope. This result indicates that increment threshold and SEM increase more slowly than base contrast and magnitude estimate, respectively. A comparison between the adapted and unadapted functions 


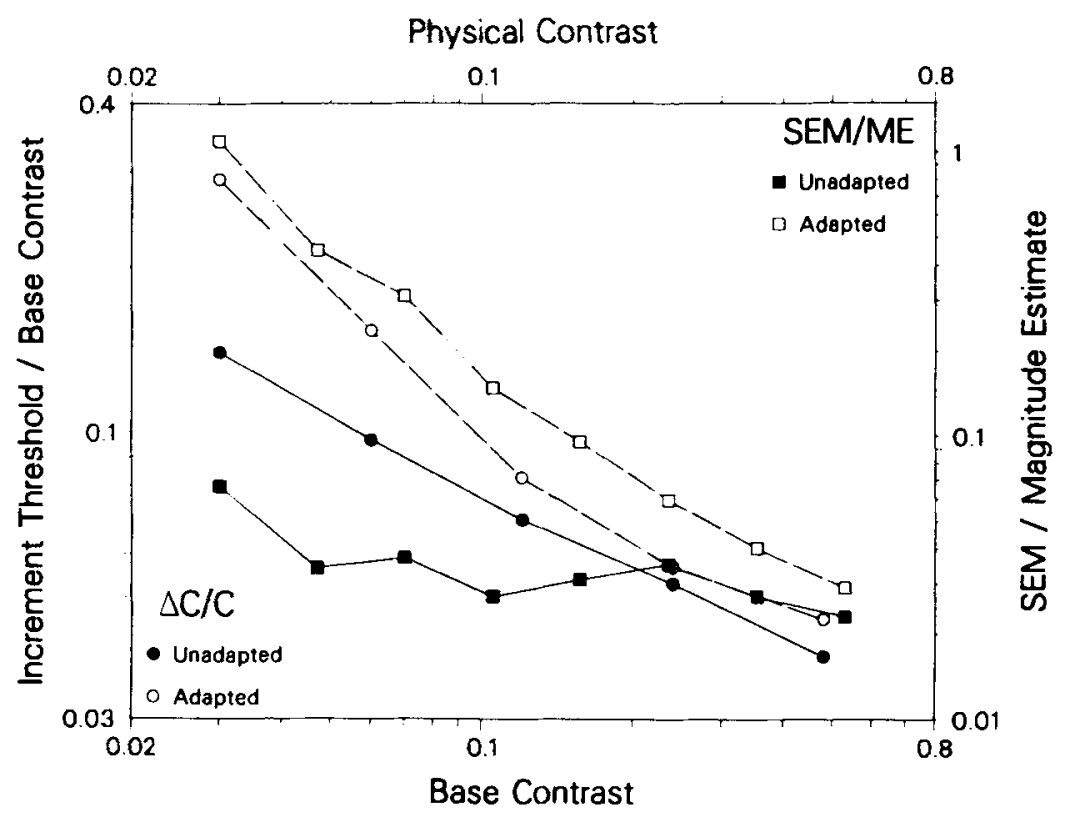

Figure 9. Relative measures of increment threshold and variability (within-subject) of magnitude estimates as a function of contrast both before and after adaptation to a highcontrast pattern. Fach data point is the mean of all spatial and temporal frequencies across all observers. Increment threshold data points (circles) correspond to the bottom and left axes, and magnitude estimation data points correspond to the top and right axes. A horizontal curve would be obtained if either of the functions obeyed Weber's law.

shows that adaptation serves to exaggerate this trend. Second, when the data are presented in this way, the effects of adaptation on both measures are qualitatively similar. Both change following adaptation, and the effects of adaptation are largest for lower contrast stimuli, and negligible at high contrasts. It is also interesting that the correlation between relative increment threshold and SEM is more evident following adaptation; this trend is apparent in both the mean and individual functions.

The implication of these findings is that resolving power for luminance contrast is determined by the system's variability, or noise, rather than by its gain. This is consistent with the conclusion reached by Legge, Kersten, and Burgess (1987), based on their measurements of contrast discrimination in the presence of externally added visual noise. Legge et al. concluded that the increase in increment threshold with increasing base contrast reflects

Table 1

Correlation Coeficients: Mean Increment Threshold/Base Contrast Versus Mean SEM/Magnitude Estimate

\begin{tabular}{|c|c|c|c|c|c|c|}
\hline \multirow{3}{*}{$\begin{array}{l}\text { Adapting } \\
\text { Contrast }\end{array}$} & \multicolumn{6}{|c|}{ Observers } \\
\hline & \multicolumn{2}{|c|}{ B.K.T. } & \multicolumn{2}{|c|}{ L.P.A. } & \multicolumn{2}{|c|}{$M$} \\
\hline & $r$ & $p$ & $r$ & $p$ & $r$ & $p$ \\
\hline $\begin{array}{l}0.0 \\
0.53\end{array}$ & $\begin{array}{l}.803 \\
.959\end{array}$ & $\begin{array}{r}.016 \\
<.001\end{array}$ & $\begin{array}{l}.634 \\
.968\end{array}$ & $\begin{array}{r}.089 \\
<.001\end{array}$ & $\begin{array}{l}.789 \\
.991\end{array}$ & $\begin{array}{r}.019 \\
<.001\end{array}$ \\
\hline
\end{tabular}

Note-Observers J.B. and L.E. each participated in only one of the experiments; individual correlations were therefore not possible. However, the data obtained from these observers were used to calculate mean correlations. an increase in the observer's internal noise. Variability in magnitude estimates of suprathreshold contrast may provide a direct measure of this internal noise. Because the relationship between noise and increment threshold is strengthened by adaptation, it is tempting to speculate that perhaps the adapted state is "normal" and that the complete absence of adaptation may be the exception rather than the rule.

\section{REFERENCES}

Barlow, H. B., Macleod, D. I. A., \& van Meeteren, A. (1976). Adaptation to gratings: No compensatory advantages found. Vision Research, 16, 1043-1045.

Biondini, A. R., d DeMATtiello, M. L. (1985). Suprathreshold contrast perception at different luminance levels. Vision Research, $25,1-9$.

Blakemore, C., Muncey, J. P. J., Ridley, R. M. (1971). Perceptual fading of a stabilized cortical image. Nature, 233, 204-205.

Bodis-Wollner, I., Hendley, C. D., \& Kulikowski, J. J. (1972). Electrophysiological and psychophysical responses to modulation of contrast of a grating pattern. Perception, 1, 341-349.

Bradley, A., Ohzawa, I. (1986). A comparison of contrast detection and discrimination. Vision Research, 26, 991-997.

Burton, G. J. (1981). Contrast discrimination by the human visual system. Biological Cybernetics, 40, 27-38.

Cannon, M. W. (1979). Contrast sensation: A linear function of stimulus contrast. Vision Research, 19, 1045-1052.

Cannon, M. W. (1984). A study of stimulus range effects in free modulus magnitude estimation of contrast. Vision Research, 24, 1049-1055.

Carlson, C. R., \& PiCA, A. (1979). Invariance in sine wave contrast discrimination. Investigative Ophthalmology \& Visual Science, 19(Suppl.), 61.

DEVALOIS, K. K. (1977). Spatial frequency adaptation can enhance contrast sensitivity. Vision Research, 17, 1057-1065. 
FeCHNER, G. T. (1912). Elemente der psychophysik. In B. Rand (Ed.), The classical psychologists (pp. 562-572) (H. S. Langfeld, Trans.). London: Constable. (Original work published 1860)

Fiorentini, A., MAFFEI, L. (1973). Contrast perception and electrophysiological correlates. Journal of Physiology, 231, 61-69.

Foley, J. M., LeGGe, G. E. (1981). Contrast detection and nearthreshold discrimination in human vision. Vision Research, 21, 1041-1053.

Franzen, O., \& Berkley, M. (1975). Apparent contrast as a function of modulation depth and spatial frequency: A comparison between perceptual and electrophysiological measures. Vision Research, 15, 655-660.

Gottesman, J., Rubin, G. S., \& LegGe, G. E. (1981). A power law for perceived contrast in human vision. Vison Research, 21, 791-799.

KoHAYAKaWA, Y. (1972). Contrast-difference thresholds with sinusoidal gratings. Journal of the Optical Society of America, 62, 584-587.

KuLIKowski, J. J. (1976). Effective contrast constancy and linearity of contrast sensation. Vison Research, 16, 1419-1431.

LEGGE, G. E. (1981). A power law for contrast discrimination. Vision Research, 21, 457-467.

LegGe, G. E., Kersten, D., Burgess, A. E. (1987). Contrast discrimination in noise. Journal of the Optical Society of America A, 4, 391404.

MANSFELD, R. J. W. (1976). Visual adaptation: Retinal transduction, brightness and sensitivity. Vision Research, 16, 679-690.

Nachmias, J., \& SANSbuRY, R. V. (1974). Grating contrast: Discrimination may be better than detection. Vision Research, 14, 1039-1042.

Stevens, S. S. (1957). On the psychophysical law. Psychological Review, 64, 153-181.

Stevens, S. S. (1958). Some similarities between hearing and seeing. The Laryngoscope, 68, 508-527.

STEVENS, S. S. (1961a). The psychophysics of sensory functions. In W. A. Rosenblith (Ed.), Sensory communication (pp. 1-33). Cambridge, MA: MIT Press.

SteVENS, S. S. (1961b). To honor Fechner and repeal his law. Science, $133,80-86$.
Swanson, W. H., Wilson, H. R., Giese, S. C. (1984). Contrast matching data predicted from contrast increment thresholds. Vision Research, 24, 63-75.

SwIFT, D. J., \& SMITH, R. A. (1984). An inherent nonlinearity in nearthreshold contrast detection. Vision Research, 24, 977-978.

WeTHERHILL, G. B., \& LEVITT, H. (1965). Sequential estimation of points on a psychometric function. British Journal of Mathematical \& Statistical Psychology, 18, 1-10.

\section{NOTES}

1. According to Stevens (1957), perceptual continua divide themselves into two general classes that roughly correspond to the traditional distinction between quantity and quality. Continua having to do with "how much," such as loudness or brightness, are believed to be mediated by an additive or prothetic process at the physiological level. Continua having to do with "what kind" or "where," such as pitch or visual position, are believed to be mediated by a substitutive or metathetic process. The two classes can be distinguished psychophysically by a number of functional criteria. Measurements of continua classified as metathetic generally confirm Fechner's assertion.

2. Swanson, Wilson, and Giese (1984) were able to construct a model that enabled the prediction of increment thresholds from contrastmatching measures of gain. However, since contrast matching provides an indirect measure of gain and the data obtained from both procedures provide an indication of resolving power (i.e., in an increment threshold procedure stimuli are adjusted to be just noticeably different along a particular dimension, and in a matching procedure variability in an observer's matches provides an indication of the just noticeable difference), a correlation between the two would be expected.

(Manuscript received November 30, 1987; revision accepted for publication June 6, 1988.) 